Est Ag 46 (2011) 313-325

\title{
A. Villarejo: Misionero en la Selva Baja del Perú 1934-1949
}

CATHERINe HeymanN*

RESUMEN: La Dra. Catherine Heymann, profesora de la Universidad de Angers, ha vivido muy unida a la vida y aventura del agustino P. Avencio Villarejo, tan conocido en la Amazonia. Lectora asidua de sus obras, sobre todo de Así Es la Selva y lo que ha dado sustento a los relatos que dieron origen a esta enciclopedia amazónica. La aventura de un agustino en la Selva, se introduce al interior del mundo de este misionero, poniendo de relieve su capacidad y valentía, recorriendo bosques y ríos de la selva baja entre 1934 y 1949. El protagonista de esta historia analiza meticulosamente las más diversas experiencias tanto de la gente que encuentra en su caminar como la diversidad en cuanto a la geografía, las plantas y las especies animales. Recorrer esta vida es una lección de coraje misionero en las distintas dimensiones, que la autora asume admirada.

PALABRAS CLAVE: misionero, Amazonia, la selva, Agustinos.

ABSTRACT: Dr. Catherine Heymann, professor at the University of Angers, has been very close to the life and adventure of the Augustinian priest Avencio Villarejo, known by this name in the Amazons. As an assiduous reader of his works, especially of the books, Así es la selva and La aventura de un agustino en la Selva, an Amazonian encyclopedia giving the background of the stories, she penetrates into the interior world of this missionary, highlights his capacity and bravery in traveling through forests and rivers of the low regions be-

${ }^{*}$ Universidad de Angers (Francia) 
tween 1934 and 1949. The protagonist of this history analyzes meticulously the most diverse experiences of the people encountered on the journey as well as the diversity of geography, the plants and the animal species. To go through this life requires a great missionary courage in all its different aspects which the author assumes admirably.

KEY WORDS: missionary, Amazons, forest, Augustinians.

La celebración del centenario de la llegada de los Padres agustinos a Iquitos, en 1901, ha sido precedida por la publicación de Memorias de un misionero. La aventura de un agustino en la Selva ${ }^{1}$ del P. Avencio Villarejo (1910-2000). Escritas al tener ya el autor ochenta y siete años, estas Memorias relatan la experiencia misionera de aquel agustino español en el mismo corazón de la Amazonía peruana, en las décadas de 1930 y 1940. Son, a un tiempo, relato de aventuras y testimonio personal. El autor reivindica claramente esta doble dimensión en sus advertencias preliminares, dirigidas "a quien se arriesgue" en su aventura, hecha de "vivencias íntimas, fruto de una estrecha relación con los selvícolas, su cosmovisión y su lenguaje”. El interés de estas Memorias nos parece múltiple tanto por la personalidad de Avencio Villarejo, especialista reconocido de la Amazonía peruana, como por el período y los lugares evocados (la parte norte de la Amazonía peruana en el segundo cuarto del siglo XX). Estas Memorias forman parte de la literatura misionera que, a lo largo del siglo XX, dio a conocer tierras lejanas o confines ${ }^{2}$. Remiten también a la literatura autobiográfica que muy a menudo le da vida al análisis del historiador. Por fin y, sobre todo, dan testimonio de las diferentes facetas de la presencia, inicialmente controvertida, y del papel llevado por los agustinos en aquella región del Perú desde principios del siglo XX.

Avencio Villarejo nació en la castellana provincia de Zamora, en 1910; estudió en Valladolid, donde emitió los votos en la Orden de San Agustín en 1925. Fue destinado a Filipinas, en 1931, viaje largo que lo llevó de Barcelona a Manila via Génova, el estrecho de Mesina, Puerto Said, Suez, Djibuti, Colombo, Belawan y Singapur; primer viaje anhelado y contemplado por él con mucha curiosidad, no exenta de algunos temores, consciente de

\footnotetext{
${ }^{1}$ Ediciones Peisa, Lima, 2000, en asociación con el CETA de Iquitos (Centro de Estudios Teológicos de la Amazonía).

${ }^{2}$ Citemos, por ejemplo, las Crónicas Akulurakeas del P. Segundo Llorente. Aquel jesuita español (1906-1989) pasó unos cuarenta años en el Círculo Polar Ártico, conviviendo con los esquimales, cerca del río Yukón. Publicó una docena de relatos sobre Alaska entre los que figuran En el país de los eternos hielos: Alaska boreal (1939) y Aventureros del Círculo Polar (1942).
} 
situarse en una "vieja tradición de navegar y navegar, desde el siglo XVI, con el padre Andrés de Urdaneta, que había abierto la ruta al mismo destino, aunque por el occidente" (p. 20) ${ }^{3}$.

Después de ordenarse sacerdote en Manila, el Superior Provincial de la Orden, A. Polanco ${ }^{4}$, lo destinó a las misiones amazónicas del Perú, creadas por los agustinos a principios del siglo XX. Fue un largo periplo de tres meses, con rumbo a Iquitos. La aventura no sólo estaba en las distancias sino también en los medios de transporte: los barcos de vapor, el tren para atravesar la Cordillera en su punto más alto ${ }^{5}$, un aeroplano incierto para llegar hasta las márgenes del Ucayali y, por fin, un hidroavión hasta la base militar situada en el Itaya (Iquitos).

Para el joven misionero, fue el principio de una estancia de catorce años en la selva, de los que pasó ocho (1934-1942) surcando ríos, único medio para visitar las poblaciones y para realizar su labor misionera. Ponía así en práctica el consejo que le dio uno de sus predecesores, el P. Lucas Espinosa: ${ }^{6}$ "Vete a los ríos, no te quedes en la ciudad. El misionar por los ríos entraña grandes dificultades y peligros pero también da satisfacción..." (p. 23). Nombrado párroco de Iquitos desde 1942 hasta 1948, siguió viajando, pero se dedicó esencialmente a su ministerio y a las obras en la misma ciudad (en particular la construcción de la iglesia catedral de Iquitos, 1944-1947).

Durante los ocho primeros años, realizó más de sesenta viajes: por el Putumayo, el Marañón, el Amazonas, el Yavarí, después por las tres parroquias de Caballococha, cerca de la frontera colombiana, y, a partir de 1942, por todo el distrito de Iquitos, que se extendía a ambas orillas del Amazonas y de sus islas y era el más poblado, debido a su proximidad con la capital. Las mayor parte de las veces, se desplazaba solo, en lancha o canoa con

\footnotetext{
${ }^{3}$ Andrés de Urdaneta (1508-1568): acompaña a El Cano en 1525 en su segunda expedición a las Islas Molucas. Regresa España y después se fue a México donde ingresó en la Orden de los Agustinos en 1553. Navegante excepcional y cosmógrafo famoso; fue llamado por Felipe II para conducir una expedición de colonización a las islas Filipinas (1564) y sobre todo encontrar un camino de regreso seguro. Revolucionó la navegación pacífica estableciendo, a partir de latitudes más septentrionales, la " Ruta del galeón de Manila".

${ }^{4}$ Agustino español (1881-1939), obispo de Teruel en 1935. Hecho prisionero en 1938, fue ejecutado por los Republicanos en 1939.

5 Es la línea de ferrocarril más alta del mundo (4.781m en el túnel de la Galera); une la costa a los Andes.

${ }^{6}$ Autor de varios trabajos lingüísticos entre ellos: Los Tupi del Oriente Peruano, Madrid, 1935; Contribuciones lingüísticas y etnográficas sobre algunos pueblos indígenas del Amazonas peruano, CSIC, 1955; en colaboración con M. Castelví, Propedéutica Etnoglotológica y diccionario clasificador de las Lenguas Indoamericanas, Madrid, Ed. Instituto Bernardino de Sahagún, 1958; Breve diccionario analítico castellano-tupi del Perú, Sección Cocama, Iquitos, CETA, 1989.
} 
remo mientras adquiría conocimientos prácticos de los cuales carecía por completo al principio.

Numerosas páginas ilustran esta vida itinerante y hacen ver, de manera muy concreta, la vida cotidiana del misionero.

La circulación en lancha exigía transporte reducido de cargas, por lo cual comía lo que se le ofrecía o lo que encontraba: pescado, aves de corral, animales de la selva (monos, caimanes, manatíes), insectos como las hormigas siquisapas (cuyo voluminoso abdomen es comestible), suris (larvas de coleópteros que viven en el tallo del aguaje, palmera de la Selva Baja), huevos de charapas ${ }^{7}$ (tortugas acuáticas), yuca asada o frita... Para el aseo, usaba un pate (típico cuenco obtenido a partir del pericarpio del tutumo) lleno con agua del río mientras que sus necesidades se cumplían al modo "ecológico", a costa de unos divertidos sustos suscitados por el medio selvático.

El estricto cumplimiento del derecho canónico planteaba problemas relacionados con el medio ambiente. El hábito, especialmente, fue un motivo de preocupación. El misionero tenía la obligación de llevar en toda circunstancia un hábito de lana negra. Además del color que aumentaba el calor y del peso (verdadero lastre en caso de naufragio), este hábito retenía el sudor y era totalmente inadecuado a los modos de vida en la selva amazónica. Tampoco era posible prescindir de la lectura cotidiana del breviario "sub peccato gravis", la cual debía hacerse después de un día remando y resistiendo las picaduras de varios insectos: ataques diurnos y nocturnos de la manta blanca (mosquito diminuto cuya picadura resulta muy dolorosa) o del isango (ácaro que vive en el campo y que provoca fuerte comezón). Reflexionar (¡muy a menudo en su carne!) sobre estos aspectos específicos, sugirió al P. Villarejo unos comentarios bien claros en sus Memorias:

"Bueno, óptimo, es rezar, ¿pero en tales condiciones?... Llego a la conclusión de que los misioneros no estábamos adiestrados para el ejercicio del ministerio y para enfrentar solos tales contingencias de la vida. Demasiada filosofía y teología; pero poco o nada de ciencias que nos aproximasen al hombre y, menos aún, de los oficios del quehacer rutinario: culinaria, carpintería, albañilería, pintura, enfermería, para las reparaciones de la casa e iglesia y otras mil tareas que demanda la práctica pastoral" (p. 52). Logró lo mismo que otros misioneros llegados de Filipinas: la autorización para usar el hábito blanco. ¡Además de la mejora física, los agustinos ganaron que ya no les apodaran igallinazos!

\footnotetext{
${ }^{7}$ Término también utilizado por los costeños para designar, de manera bastante condescendiente, a los habitantes de la Selva.
} 
Una parte importante de sus Memorias da cuenta de su labor misionera. En esto, A. Villarejo siguió escrupulosamente el segundo de los consejos dados por el P. Espinosa: “... Lleva un cuaderno para anotar los sacramentos que administres, para luego transcribir las partidas correspondientes a cada circunscripción" (p. 24). Además de llevar cuentas muy precisas, elabora una ficha recapitulativa de todas sus actividades con las poblaciones ribereñas (mestizas o indígenas). Logró las siguientes cifras para el periodo 1935-1942: 205 pueblos y caseríos visitados; 759 charlas a adultos; 1.066 horas en escuelas; 2.881 bautismos de párvulos; 292 bautismos de adultos, 3.506 confirmaciones, 856 confesiones, 1.204 comuniones, 130 matrimonios religiosos; 6 matrimonios civiles. Termina esta síntesis, subrayando que tales cifras fueron ampliamente superadas (en lo que se refiere a la administración de sacramentos, sermones, enseñanza en las diferentes escuelas de las que estuvo encargado) en los siete años que pasó en la ciudad de Iquitos (1942-1949).

Es muy reveladora esta contabilidad itinerante. Da parte de la constancia de su presencia "en el terreno" y explica la cantidad de observaciones que pudo hacer durante estas visitas: desnutrición, enfermedades graves y contagiosas (lepra, uta $^{8}$, parasitosis, paludismo), comercio inicuo de los regatones, consciencia de la explotación de los indios por patrones blancos poco escrupulosos ("¿Qué podía hacer el misionero ante tan descarado agiotaje?" p. 55), aunque se niega a generalizar esta visión y defiende a patrones paternalistas (p. 56), con evidente conciencia de los límites del sistema misionero en ciertos casos (pp. 82-83) ${ }^{9}$. Revela también el importante papel desempeñado por estos misioneros en la voluntad política de integrar la Amazonía en el territorio nacional, en la primera mitad del siglo XX.

A finales del siglo XIX, la Amazonía peruana fue una de las piezas importantes del proyecto político y económico, de inspiración positivista, establecido por dirigentes que querían hacer del Perú un verdadero Estadonación. Tal ambición suponía el control de todo el territorio, la integración al modelo nacional de las poblaciones indígenas, designadas como las responsables del retraso del país y la explotación de las riquezas naturales, de las cuales el caucho amazónico fue, sin lugar a dudas, la más cotizada.

\footnotetext{
${ }^{8}$ Lepra blanca.

${ }^{9}$ Relatando la experiencia del P. Ismael Barrios, su sucesor en la misión del Napo en 1945-1946, y el primer contacto de éste con los indios "vacacochas", apartados hasta entonces de la penetración misionera, evoca A. Villarejo las dudas del Padre a la hora de administrar los sacramentos (p. 82), sin olvidar los problemas planteados por la utilización de varios idiomas: del castellano al quechua, del quechua al záparo. Comenta al respecto el P. Avencio: "A tanta distancia cultural, con un pensamiento que en nada compatilizaba con nuestro modo de ver el mundo, ¿qué más se podía hacer?” (p. 83).
} 
Por otra parte, la Santa Sede, afectada por las reformas liberales que habían conocido la mayor parte de los países de Europa y por la pérdida de los Estados Pontificios, se esforzaba por definir una estrategia que le permitiera encontrar nuevas tierras de misiones y, en el caso de América Latina, recuperar lo perdido después de luchas encarnizadas con las jóvenes Repúblicas ${ }^{10}$. Por eso, durante su pontificado (1878-1903), León XIII favoreció una nueva expansión de las actividades misioneras. La Iglesia peruana elaboró un discurso progresista que hacía de la religión católica un elemento esencial de la nacionalidad peruana y un instrumento imprescindible para forjar la unidad del país. Las misiones eran el medio para integrar a las poblaciones indígenas: se trataba de ir "en pos del indio errante, con el único objeto de hacerlo hombre civilizado, cristiano y también peruano"11.

Consecuencia de ello fue un proyecto relativo a la creación de tres prefecturas apostólicas en territorio selvícola, confiadas a religiosos europeos, presentado al ejecutivo peruano, quien lo aprobó en octubre de $1898^{12}$. Los argumentos aducidos para el establecimiento de misiones evangélicas, en estos territorios descubren claramente los objetivos del gobierno: “... que las numerosas tribus que habitan en esa parte de la República se hallan en estado de barbarie, manteniendo inexplorada gran parte de aquella región, e impidiendo el establecimiento de pobladores civilizados con las industrias y mejoras consiguientes..."13.

La Prefectura de San León del Amazonas (la mayor) le toca a los agustinos españoles ${ }^{14}$. La acogida que les fue reservada (marzo de 1901) resultó bastante mitigada. El coronel P. Portillo, prefecto civilista, saludó la llegada a Iquitos del P. Paulino Díaz, primer Prefecto Apostólico con estas palabras:

\footnotetext{
${ }^{10}$ Sobre la cuestión religiosa en el Perú contemporáneo, véase Pilar García Jordán: Iglesia y poder en el Perú contemporáneo, 1821-1919, Cusco, CBC, 1991 y "Régimen eclesiástico de principios de siglo en la Amazonía peruana", Kanatari, $\mathrm{n}^{\circ} 850$, Primer Centenario del Primer Vicariato Apostólico, Diciembre 2000, Iquitos, pp. 23-26.

11 Art. cit., p. 23.

${ }^{12}$ Se trataba de la de San León del Amazonas -en la parte norte de la Selva- que se extendía hasta las fronteras de Brasil, Colombia y Ecuador, con sede en Iquitos; la segunda -San Francisco de Ucayali-ocupaba la región central, con sede en Santa Rosa de Ocopa;la última -Santo Domingo de Urubamba- se extendía hasta la frontera boliviana y tenía su centro en el Cusco.

13 Id., p. 25.

${ }^{14}$ Sobre las misiones en la Amazonía peruana, léase Pilar Garcí Jordán, Cruz y arado, fusiles y discursos. La construcción de los Orientes en el Perú y Bolivia, 1820-1940, Lima, IFEAIEP,2001,pp. 197-246 ; P. Joaquín García Sánchez, "Un siglo de presencia de la Iglesia en Iquitos", Kanatari, n 799, Iquitos, enero 2000, pp. 45-50 y "La Prefectura Apostólica de San León del Amazonas (antecedentes)", Kanatari, Primer Centenario..., nº 850, diciembre 2000, Iquitos, pp. 7-12; P. Avencio Villarejo, Los Agustinos en el Perú y Bolivia, Lima, ed. Ausonia-Ceta, 1965 .
} 
"le felicito por su feliz arribo y me felicito por la civilización que Ud. se propone difundir entre las tribus bárbaras"15. La formulación definía nítidamente la función y el área geográfica de las tareas que se deseaban ver ejercer a los misioneros. Por otra parte, a la burguesía comerciante local le humillaba que se considerase a "la capital peruana del caucho" como un territorio de misiones.

Encontramos varias huellas de esta situación en las Memorias de A. Villarejo. En su advertencia preliminar, que sirve también para precisar el marco histórico, describe la situación religiosa en estos términos. "...la atención religiosa había descendido a niveles bajo cero, con unos cuantos curas doctrineros ${ }^{16}$ involucrados en la vorágine del caucho, como medio de subsistencia" (p. 14). El P. Avencio retoma aquí los principales reproches hechos por los agustinos al clero local (vida disoluta, ausencia de vocación, afán de lucro). La frase citada remite al conflicto de jurisdicción que brotó entre, por una parte, las autoridades civiles y eclesiásticas y, por otra parte, las prefecturas apostólicas. Varios "curas doctrineros" se negaron a depender de la autoridad jurídica de la nueva Prefectura; fueron suspendidos pero algunos no aceptaron el castigo y se mantuvieron en sus cargos. Uno de los argumentos de los que se valían era su condición de "nacionales" frente a misioneros extranjeros. Como historiador de la misión, A. Villarejo enumera algunos de los enemigos de los agustinos de la primera hora (p. 25). Subraya también la falta de entusiasmo de la burguesía loretana que, dice: "tomó a los religiosos como censores de sus vicios" (p. 14) y la hostilidad de la población local a la "que repudiaba la idea de misiones consideradas como evangelizadoras de salvajes" (ibid.).

Cuando A. Villarejo llegó a Iquitos en 1934, es decir en plena depresión económica, la resistencia a los agustinos no había terminado por completo, aunque ya no tomaba la forma de panfletos o manifestaciones callejeras ( $\mathrm{p}$. 23). Subsistía un fondo de hostilidad hacia los religiosos "que no edificaban obras para la mejora de la ciudad y que no recorrían los ríos en misión"(p. 25). En cuanto al segundo punto, confirma A. Villarejo el relativo abandono

${ }^{15}$ Extracto de los Archivos del Vicariato de Iquitos, citado por el P. García Sánchez, $K a$ natari, diciembre 2000, p. 11.

${ }^{16} \mathrm{El}$ subrayado es nuestro. Creadas en tiempos de la Conquista, estas doctrinas fueron pensadas para responder a las peculiaridades de la evangelización de los indios. Se basaban en la atribuciones de una parte de territorio a un doctrinero que estaba encargado de la evangelización de sus habitantes. Muy a menudo, se desviaron de sus objetivos primitivos y pasaron a tener un carácter netamente económico. 
de las poblaciones ribereñas debido a las dificultades de comunicación y la falta de medios humanos y financieros ${ }^{17}$.

Es preciso añadir las dificultades vinculadas al funcionamiento de la Misión agustina de Iquitos a la que la Santa Sede no mandó Vicario General desde 1935 hasta 1942. El nombramiento de Monseñor García Pulgar como Vicario Apostólico en 1942 puso fin a esta acefalía y coincidió con las nuevas responsabilidades que le tocaron a A. Villarejo en Iquitos. Observa entonces un sosiego progresivo de los espíritus ${ }^{18}$.

La situación de inestabilidad en las fronteras del Estado (con Colombia y Ecuador) fue otra fuente de roces entre la Misión y el Gobierno, que mandó expulsar inclusive a tres misioneros agustinos, juzgados "extranjeros indeseables" en el territorio nacional ${ }^{19}$.

Desde la Independencia, tanto Colombia como Ecuador no dejaron de reivindicar un acceso directo al Amazonas. Colombia tenía acceso al gran río a través del Caquetá pero tenía que pasar por Brasil; Ecuador a su vez poseía la vía del Napo, pasando por el territorio peruano. La situación no era nada satisfactoria para ninguno de los dos países y revela la ausencia de una verdadera política de integración en las primeras décadas del siglo $\mathrm{XIX}^{20}$.

Con la firma del Tratado Salomón-Lozano, Colombia obtuvo del Perú más de $113.000 \mathrm{~km} 2$, situados ente el Caquetá y el Putumayo, así como el "Trapecio amazónico", de modo que en 1930 el puerto peruano de Leticia pasó a ser territorio colombiano ${ }^{21}$. La población del Loreto sintió este tratado como una mutilación y un signo de sumisión a las autoridades norteamericanas $^{22}$. En septiembre de 1932, un grupo de habitantes del Loreto se apoderó del puerto de Leticia. El incidente, después de haber sido sometido

17 Pilar García Jordán estableció un cuadro del número de misioneros en las tres prefecturas durante el periodo 1907-1930. Para el conjunto de la prefectura de San León del Amazonas, oscila la cifra entre seis y nueve misioneros. Cruz y arado..., p. 223.

${ }_{18}$ Para ampliar la iglesia Matriz y convertirla en catedral, recaudó fondos privados y recurrió a la colaboración popular. Las obras fueron acogidas de manera favorable. Cesaron las disensiones hasta el punto de que menciona la contribución financiera de los masones de Iquitos.

${ }^{19}$ Se trata de los P. Lucas Espinosa, P. Donato Gorrochátegui y P. Valeriano Rivero.

${ }^{20}$ Sobre los conflictos fronterizos con Ecuador y Colombia, léanse H. Morey Alejo y G. Sotil García: Panorama histórico de la Amazonía peruana, Ed. Municipalidad provincial de Maynas, Iquitos, Perú, octubre 2000 (cap. 7 y 9); M.C. Ríos Zañartu: Historia de la Amazonía peruana (compendio), El Matutino, Iquitos, 1995, pp. 132-135.

${ }^{21}$ Fue suscrito secretamente en 1922; lo aprobó el Congreso peruano en octubre de 1927 y fue firmado por el presidente A. Leguía, acusado de entreguismo, en enero de 1928.

${ }^{22}$ La cesión de esta parte del territorio peruano a los colombianos fue ideada por los norteamericanos como una compensación por la pérdida de la región de Panamá (que formaba parte inicialmente de Gran Colombia), convertida en Estado de Panamá, en 1903, con 
a la S.D.N., se resolvió pacíficamente en junio de 1933 por un Acto que especificaba la salida de los peruanos de Leticia y la retirada de las fuerzas militares de los dos bandos ${ }^{23}$.

En junio de 1935, por orden del Prefecto del Departamento, A. Villarejo se vio encargado de "visitar" al Putumayo en todo su recorrido. Tal viaje lo pagaba el Estado, que le confiaba el trabajo de establecer un informe sobre la situación social y religiosa de dicha zona, después de la toma de posesión por Colombia de la margen izquierda del río y de una parte de la derecha. Esta tarea testimonia la importancia de la presencia misionera en la ocupación del territorio nacional. Durante este viaje, A. Villarejo visitó los lugares en los que la Casa Arana había extendido su imperio, reduciendo las poblaciones indígenas a una explotación descarada y dejando que se cometieran las peores atrocidades, el episodio más sangriento de la historia del caucho americano, conocido como "escándalo del Putumayo" (1903-1914) ${ }^{24}$. Ante la inminencia de la ratificación del tratado Salomón-Lozano, J.C. Arana había desplazado las poblaciones indígenas (huitotos, boras, andoques) hacia la margen derecha del Putumayo, región muy insalubre, en la que muchos indios perecieron. El P. Avencio recalca la miseria de la zona, desde el punto de vista económico y religioso (así se explica, en el segundo caso, el número elevado de ciento dieciocho bautismos administrados con cierta urgencia, en un solo día, en El Estrecho, p. 29).

Realizó el más largo de sus viajes por el Napo en 1941, en un marco estrictamente misionero. Recorrió por cinco meses este río, cuyas cabeceras se encuentran en los Andes ecuatorianos ${ }^{25}$. Concluyó este largo recorrido de

miras a la construcción del canal. En cambio, el Perú recibiría el territorio de Sucumbíos, cedido por Ecuador a Colombia antes de la firma del tratado Salomón-Lozano. En 1942, durante otro conflicto territorial, el Perú devolvió Sucumbíos a Ecuador.

${ }^{23}$ En marzo de 1933, las fuerzas colombianas del Alto Putumayo atacaron la guarnición de Güeppí, respondiendo a la ocupación de Leticia. Varios soldados peruanos murieron en el ataque, entre ellos el Sargento Lores (1906-1933), héroe nacional de este conflicto, que se resolvió diplomáticamente en mayo de 1934.

${ }^{24}$ Sobre las etapas de la conquista de esta zona, en particular, económica, léase A-M. d'Ans, L'Amazonie peruvienne indigène, Paris, Payot, 1982, pp. 100-226. Sobre el "escándalo del Putumayo", Pilar García Jordan, "En el corazón de las tinieblas... del Putumayo 1890-1932. Frontera, caucho, mano de obra indígena y misiones católicas en la nacionalización de la Amazonía", Revista de Indias, Vol. LXI, sept-dic 2001, n²23, Dossier La Amazonía Andina (P. García Jordán coord.), pp. 591-617.

${ }^{25}$ Desde 1840, no dejaron de pelearse Ecuador y el Perú la posesión de Tumbes, Jaén y Maynas. Varios incidentes tuvieron lugar tanto en la Costa como en los puestos de vigilancia situados a lo largo de la frontera amazónica (combates de Angoteros en 1903, de Torres Causano en 1904). La diplomacia-arbitraje del rey de España, mediación de Argentina, Brasil y Estados Unidos no encontraron soluciones satisfactorias de modo que siguieron las tensiones hasta el episodio del ataque de Rocafuerte. 
unos $800 \mathrm{kms}$ con la visita de la guarnición peruana de Cabo Pantoja, situada en la frontera peruano-ecuatoriana, cerca de la confluencia del Napo y del Aguarico ${ }^{26}$. Estas visitas a las fronteras recalcan el carácter "geopolítico"27 de la actividad misionera a lo largo del siglo XX, en una Amazonía peruana que se ofrecía como un territorio "vacío" y rico de recursos naturales ${ }^{28}$. Quisieron las circunstancias que un día después de su estancia en Cabo Pantoja y mientras visitaba otra guarnición situada en el Curaray, estallaran hostilidades entre la guarnición de Cabo Pantoja y la de Rocafuerte (Ecuador). La "toma de Rocafuerte" por los peruanos fue la acción más importante de una serie de operativos llevados a cabo en esta porción de frontera amazónica.

El relato del P. Avencio describe estos enfrentamientos sangrientos y realza la determinación patriótica de ambos bandos. Detalla primero la topografía de los lugares y la ubicación de ambas guarniciones ${ }^{29}$ y subraya a continuación el carácter absurdo de estos combates.

Las visitas de A. Villarejo a las fronteras del Estado peruano no terminaron, todas, de manera tan trágica. Contribuyen al recuerdo de lo que fue la explotación del caucho y sus consecuencias humanas y económicas así como dejan memoria de la vida de estos puestos militares fronterizos ${ }^{30}$, siempre ilustrando la participación de los misioneros en la afirmación de la soberanía nacional.

\footnotetext{
${ }^{26}$ Una de las explicaciones sobre la expulsión del P. Espinosa sería el haber pasado de la guarnición peruana a la ecuatoriana sin pedir la debida autorización a las autoridades militares peruanas. Menciona también el P. Avencio el caso del P. Díez expulsado menos de dos años después de su llegada por haber criticado, en voz alta, a los militares que mantenían "una sensibilidad a flor de piel contra cualquier atisbo que pudiera herir el hondo sentimiento popular nacionalista" (p. 38).

${ }^{27}$ Pilar García Jordán, La cruz y el arado..., pp. 436-442.

${ }^{28}$ Cuenta A. Villarejo en sus Memorias que, en la edición de 1943 de Así es la Selva, dejó constancia de unas particularidades en la zona del Alto Corrientes (en el Huandoyacu): tierra negra, barrancos con estratos de estructura y coloridos diversos y hasta rocas en formación. No le dio más importancia. Unos años más tarde, cuando era párroco de Iquitos, llegó un norteamericano que, después de haber leído su libro, quería ir a esta zona. Lo ayudó a preparar su viaje. Cuando volvió el norteamericano, le enseñó un trazado preciso del curso de la quebrada pero no dejó que el P. Avencio lo copiara. Era "Secreto de su compañía!". Quince años más tarde, brotaba el petróleo del pozo Corrientes XI (p. 96).

${ }^{29} \mathrm{Al}$ final de las Memorias, figuran diecisiete fotografías de las que varias presentan particular interés documental. Una en particular representa "el pasadizo entablado con sus respectivas garitas a cada lado, designado con el pomposo título de Puente Internacional" que indica la frontera. Estas reproducciones le permiten al lector mejor captar la realidad y la especificidad de estos puestos militares en el medio amazónico.

${ }^{30}$ En 1995 tuvo lugar un nuevo episodio sangriento entre Ecuador y el Perú, en el río Cenepa.
} 
A sesenta años de distancia, el Padre Villarejo confiesa "a media voz" (p. 56) que en sus primeros viajes, llevaba una pistola por si tenía que enfrentarse con algunos monstruos: salvajes, fieras o animales ponzoñosos, según la imagen que se había forjado de la selva a partir de sus lecturas. La realidad fue muy distinta y sus Memorias ponen de realce el resultado de estos descubrimientos y aprendizaje de las realidades amazónicas, por impregnación diaria. Lo que más tarde fuera la materia trabajada de un libro fundamental Así es la selva ${ }^{31}$, corresponde a una experiencia concreta.

Siguió otro consejo del P. Espinosa: "Lleva un cuaderno donde vayas anotando ríos y afluentes, plantas medicinales o útiles para otros fines; tipos de casa, datos étnicos de tribus y sus idiomas; haz croquis de mapas con localización de caseríos o pueblos"(p. 24). Su actitud hacia el medio natural y humano estuvo basado sistemáticamente sobre la observación, la comprensión de los fenómenos o de los comportamientos y la adaptación. Los verbos que conforman la síntesis de aquella experiencia remiten todos al conocimiento y enmarcan su trayectoria en una tradición misionera en la que el religioso se hace también historiador, etnógrafo, botanista...

A través de la relación de sus innumerables viajes, se encuentran dibujadas la topografía y la toponimia fluviales de una parte de la Amazonía peruana (en particular en los capítulos 6, 7, 8 y 9). Están puestas de relieve también, con términos sencillos, las características fenomenales de los ríos amazónicos (erosión de las márgenes, cambios frecuentes del thalweg, modificación incesante del paisaje). La descripción de las transformaciones del curso del Amazonas en Iquitos, en los años treinta y cuarenta constituye un ejemplo muy concreto de tales fenómenos: erosión de la orilla y derrumbes que erosionaron el Malecón y varios edificios, así como la modificación del canal de navegación (pp. 71-72). La conciencia de las fluctuaciones incesantes del curso del río le hace escribir al Padre Villarejo que su porvenir es un enigma. ${ }^{32}$ En el relato abundan los detalles que ayudan a comprender que tiempo y distancia se miden no en kilómetros u horas sino en bajadas y surcadas, meandros y curvas; a lo que hace falta añadir lo que llamaremos la flema amazónica que hace que un transeúnte fluvial interrogado sobre la proximidad de tal o cual lugar suele contestar: ¡ahí no más! (p. 92).

\footnotetext{
${ }^{31}$ La primera edición de esta monografía dedicada a la Provincia del Bajo Amazonas y después a toda la Amazonía peruana, es de 1943. Se publicó cuando estaba terminándose el "año amazónico" que celebraba el cuarto centenario del descubrimiento del Amazonas por Orellana y cuando se abría una exposición misionera sobre Amazonía en el Colegio San Agustín de Lima. Se compone de cinco partes: geográfica; botánica y zoológica; etnográfica; geografía política y estadística demográfica; presentación de las tribus más importantes con cuadros relativos a la enseñanza y la ubicación de los centros educativos.
} 
Se nota la misma riqueza en la observación de la flora y la fauna. El testimonio de A. Villarejo hace hincapié en la abundancia de especies ictiológicas: bufeos (delfines) de tres metros de largo, paiches de sabrosa carne, atasco de caimanes que impiden la progresión de las canoas (en una época en la que la exportación y el tráfico de pieles no habían diezmado las orillas); serpientes impresionantes como la yacumama, boa gigante de doce metros de largo y treinta centímetros de diámetro, como la vista en Caballococha, la naca-naca, víbora muy ponzoñosa que, algún día, visitó la huerta parroquial; flora útil como el ojé, cuya leche se utiliza para luchar contra el parasitismo intestinal, la yarina, el huacapú y la pona ${ }^{33}$, que proporcionan materiales naturales para la construcción de viviendas (la del subteniente de la guarnición de Cabo Pantoja), o simplemente bella para la observación como la victoria regia (nenúfar gigante) con sus hojas de un metro o dos de diámetro y sus flores sobre las que se posan los pájaros cuando buscan comida.

Las actividades misioneras del P. Avencio, de las que la educación religiosa y la educación profana fue el eje principal, se desplegaron hacia las poblaciones ribereñas. Los diferentes capítulos de las Memorias encierran el nombre de las poblaciones que él visitó: cocamas, yaguas y ticunas de la parroquia de Caballococha; secoyas, orejones y arabelas de la cuenca del Napo, incluyendo pequeños grupos de quichuas procedentes del Alto Napo ecuatoriano, llevados por patrones peruanos para explotar las riquezas de la selva (p. 75); cocamas y omaguas de la cuenca del Marañón a los que define como "plenamente adaptados a la civilización" (p. 89), con pequeños grupos de quichuas-alamas dispersos por los ríos Tigre y Corrientes, en contacto con la cultura dominante. Además de los sacramentos, las lecciones de catequesis y la mejora del ordinario de las celebraciones religiosas (comuniones, procesiones de Semana Santa) en las zonas selvícolas, uno de los medios empleados para conocer y penetrar la sicología de la gente de la Selva fue la organización de "tertulias", lugar de intercambios sobre los temas más cotidianos: medicinas populares, tradiciones, historias locales, leyendas, prácticas de "brujos" y otros "curanderos".

Una evidencia corresponde a los comentarios críticos sobre las carencias de la formación de los misioneros enviados a la Amazonía peruana sin preparación: la formación pragmática desarrollada en los campos más di-

\footnotetext{
${ }^{32}$ Para un análisis de la situación actual del curso del Amazonas en Iquitos, léanse $\mathrm{N}$. Bernex de Falen y P. García Sánchez, El río que se aleja. Cambio del curso del Amazonas, $2^{\text {a }}$ edición (corregida y aumentada), Iquitos, CETA-IIAP, 1994.

${ }^{33}$ La yarina y la pona son variedades de palmeras; el huacapú es un árbol cuya madera es muy dura.
} 
versos. La experiencia más rica fue indudablemente la relación humana, nacida del contacto con las poblaciones indígenas aculturadas o en vías de aculturación, a las que evocaba años más tarde con mucha emoción (p. 34). Toda la diversidad étnica, lingüística y cultural de una parte de la Selva peruana aparece en estas Memorias, al mismo tiempo que queda clara la voluntad de "civilizar" a las comunidades indígenas ${ }^{34}$.

Al poner en perspectiva el conjunto de la vida y de los trabajos de Avencio Villarejo, estas Memorias muestran claramente la intensidad de su labor misionera e informan sobre la materia bruta que iba a plasmar después en sus obras científicas. Revelan también su participación en la realización de un proyecto político y económico, cuyo objeto era tanto la afirmación de la soberanía nacional como la integración definitiva del Oriente peruano (y de sus poblaciones) al resto de la nación ${ }^{35}$. Por fin, estas Memorias son una parte de la "verdad" del Padre Villarejo, puesta al servicio de la escritura de la historia de los agustinos en la Amazonía peruana, contada con la manera metódica, militante y sin efectos retóricos que caracteriza a su autor.

${ }^{34}$ En su introducción a La Selva y el Hombre (1959), adaptación de su tesis de doctorado en geografía (cuyo título era Antropocosmología amazónica) da una explicación detallada y reveladora del uso que hace de los términos "civilizado, semicivilizado, salvaje y patrono" (p. 11). Por otra parte, sitúa sus Memorias en el marco de la continuación de la labor misionera que, a partir del siglo XVI, originó los primeros textos que al tratar del descubrimiento (y de la evangelización) de los indígenas (p. 136), los hicieron entrar en el tiempo histórico, ya que, para él, las condiciones climáticas hacen casi imposible el conocimiento de una "prehistoria" indígena (p. 135).

${ }^{35}$ La edificación de la Catedral de Iquitos es una clara manifestación de ello. El P. Avencio cumplió con lo que estimaba ser de su incumbencia y dotó a la capital de la Amazonía peruana de una iglesia destinada a hacer de ella "un bastión señero de peruanidad y catolicismo" (dedicatoria del autor en La Selva y el Hombre). Añadamos que las obras que mandó realizar para el adorno del interior de la Catedral son un testimonio de la vida artística iquiteña a mediados del pasado siglo, al mismo tiempo que se inscriben en una dinámica de la historia misionera. Citaremos, en particular, el tríptico sobre la pared del ábside, pintado por el afamado pintor loretano César Calvo de Araújo (1914-1970). Representa la alegoría de un misionero jesuita viajando por el Nanay en el siglo XVI; otra alegoría representa a la Virgen sobre el Amazonas con la torre de la iglesia matriz que emerge de la selva mientras que el tercer mural muestra a un misionero (representado bajo las facciones de A. Villarejo) que está hablando a la muchedumbre congregada frente a la Catedral. 